\title{
Single Molecule Microscopic Detection of Corrosion Reactions using “Turned-on" Fluorophores
}

\author{
Anuj Saini ${ }^{1}$ and Lydia Kisley ${ }^{2}$ \\ ${ }^{1}$ Case Western Reserve University, Mayfield Heights, Ohio, United States, ${ }^{2}$ Case Western Reserve \\ University, United States
}

\section{Introduction}

Prevention of corrosion is multimillion dollar problem and a major topic of interest for scientists and researchers [1]. It has been a subject of general chemistry courses with schematics showing transfer of electron from anode to cathode with the dissolution of metal oxide at the anode (Fig. 1A). Despite vast experience with corrosion, we have been unable to visualize it at the molecular scale.

Molecular level imaging of in situ corrosion has been limited due to the spatial and temporal limits of current microscope techniques. There have been efforts to obtain nanoscale resolution images of corrosion using electron microscopy, scanning electrochemical microscopy, AFM and STM. However, the dynamic information is lost because these techniques are ex-situ or do not allow for aqueous conditions. In-situ AFM and optical microscopies such as Raman or confocal allow for conditions of aqueous corrosion but, at best, hypothetical submicron resolutions have been reported with these techniques.

Fluorescence microscopy is an ideal way to detect in-situ corrosion at the molecular scale. Individual fluorescent molecules present in a sample are illuminated using a microscope with a coupled laser. A highlysensitive camera detects the fluorescence signal from the individual molecules after the separation of the emitted red-shifted fluorescence. Desired chemical process can be studied by selecting fluorophores that undergo specific fluorogenic reactions (Fig. 1B) [2]. Heterogeneities hidden in the conventional experiments can be determined by observing one molecule at a time [3]. Millisecond time resolutions and sub-diffraction limited, three-dimensional spatial resolutions at $\sim 10 \mathrm{~nm}$ can be achieved in-situ for single molecules [4]. Previous fluorescence imaging of corrosion has been limited in spatial and temporal resolutions due to the limitations in the microscopy hardware employed [5]. Photon yield to detect corrosion at a single-molecule level under realistic conditions could be increased by using state-of-the-art equipment.

We demonstrate that fluorophores "turned-on" by corrosion of iron can be detected at the single-molecule scale [6]. First, we study the properties of the dye resazurin, which becomes fluorescent in the reduced form as resorufin, at the ensemble level. Resorufin "turn-on" detects the corrosion of iron catalyzed by $\mathrm{NaCl}$ both as an observational color change and quantified increase in fluorescence emission. We also detect resorufin "turned-on" by corrosion of iron at the single-molecule level with a home-built total internal reflection fluorescence (TIRF) microscope that has significantly improved hardware capabilities as compared to previous works.

\section{Results and Discussion}

Ensemble measurements in bulk: Resazurin "turns-on" to resorufin in the presence of iron An 18-fold increase in the fluorescence signal of resazurin/resorufin in the presence of iron and $\mathrm{NaCl}$ is observed over 24 hours (Fig. 2A). The rate of change of fluorescence signal is a measure of the rate of corrosion of iron under the assumptionthat the iron particles remain electrically neutral at all times. The change in the total fluorescence signal $(565 \mathrm{~nm}-700 \mathrm{~nm}$ ) of resazurin/resorufin in the presence of $3.6 \mathrm{M}$ iron (or $2 \mathrm{~g}$ Fe per $10 \mathrm{~mL}$ of solution) at different $\mathrm{NaCl}$ concentrations was measured.

Fluorescence quantifies the increase in the rate of reaction of the corrosion by addition of $\mathrm{NaCl}$ as a catalyst Resazurin "turn-on" can detect the increase in the rate of corrosion in the presence of $\mathrm{NaCl}$. A higher rate of reaction is observed as the corrosion reaction is catalyzed by the chloride ions. The fluorescence signal increases by 1.5 -fold during the first four hours when no $\mathrm{NaCl}$ is present, whereas it increases by almost three- 
fold in the presence of $300 \mathrm{mM} \mathrm{NaCl}$ (Fig 2B). Increasing concentration of $\mathrm{NaCl}$ results in a faster increase in the fluorescence signal.

Real-time single molecule detection of "turn-on" events by corrosion by TIRF microscopy

A home built wide field TIRF microscope with $561 \mathrm{~nm}$ laser excitation, 1.49 numerical aperture with 100X magnification objective, and a scientific CMOS detector was used to overcome the previous limitations of microscopic fluorescence corrosion detection. An aqueous of solution of resazurin $(100 \mathrm{nM})$ in $300 \mathrm{mM} \mathrm{NaCl}$ was introduced to a flow cell that had iron powder particles on a microscope coverslip. Dye "turn-on" events were monitored over time as corrosion of iron progressed. The diffraction-limited single molecule fluorescence emission pattern were localized with a super-resolution fitting routine. The number of "turn-on" events observed over $2 \mathrm{~min}$ of a $155 \mu \mathrm{m} \times 155 \mu \mathrm{m}$ region of interest were counted and plotted as total single molecule events per unit area during the time elapsed from $0-4 \mathrm{hrs}$ (Fig. 2C). A steady increase in the total number of single molecule events per unit area is observed representing a constant reaction rate. The increase in the number of detected molecules over time showed some heterogeneities that hints toward a deviation from the linear model as seen in the ensemble measurements represented in Figure 2C.

\section{Conclusions}

We demonstrate that corrosion reduction reactions can be sensed at the ultimate limit of detection - the singlemolecule scale. Resazurin can "turn-on" to resorufin to detect cathodic electron transfer reactions during the corrosion of iron. Individual resorufin molecules are detected with a wide field TIRF microscope consisting of laser excitation, a high numerical aperture objective, and a high quantum yield camera. After considering the incorporation of additional components to address the diffusion of dye molecules, "turn-on" fluorescence of resazurin using single molecule spectroscopy can be a valuable tool to study corrosion of iron at the molecular level and with improved time-scales.

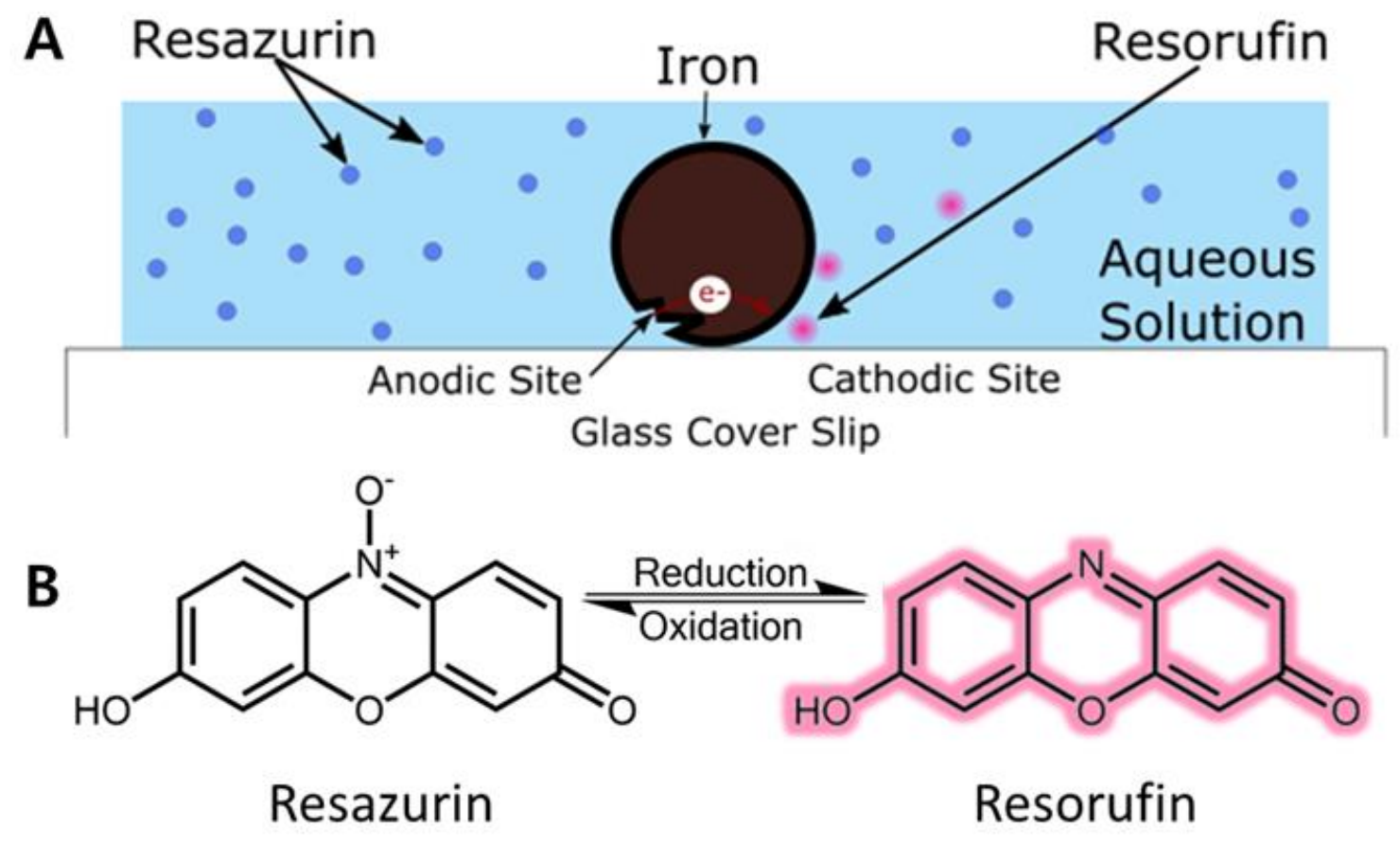

Figure 1. Schematic of "turn-on" fluorophores being detected at the interface of Fe particles. (A) Resorufin molecules (in pink) detect the interfacial cathodic sites of corrosion. (B) Resazurin undergoes reduction reaction to "turn-on" to produce the fluorescent product, resorufin. 

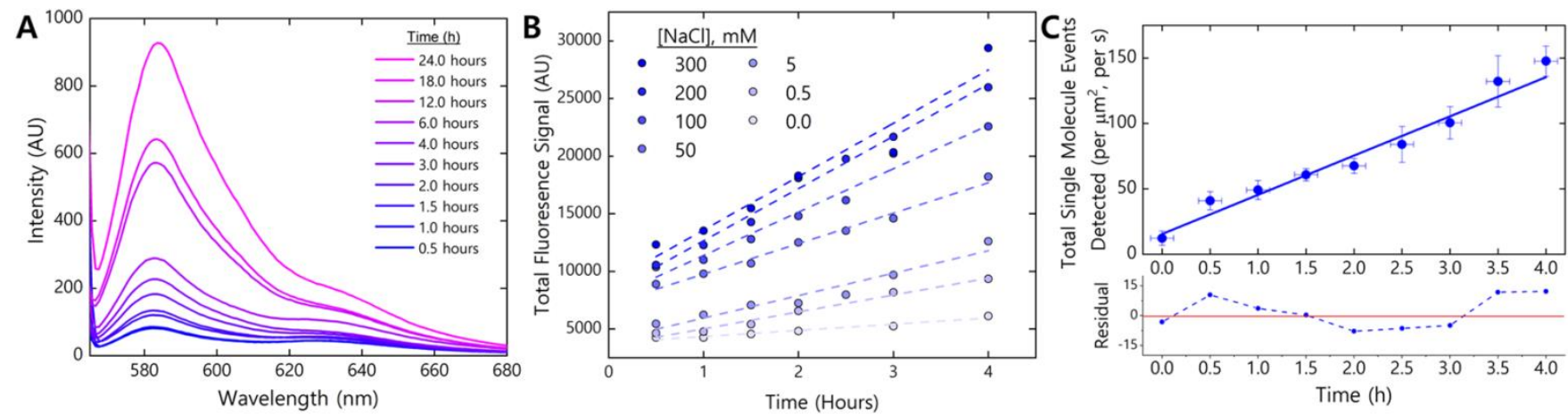

Figure 2. Real-time detection of iron corrosion by "turn-on" of resazurin molecules. (A) 18-fold increase in fluorescence signal of resazurin over 24 hours. (B) Effect of $\mathrm{NaCl}$ on the rate of increase of fluorescence. (C) Detection of resazurin "turn-on" at the single-molecule level. Quantitatively, the residuals of a fit to the data are non-random around zero, supporting that a linear relationship may no longer be a reasonable assumption at the molecular level.

References

[1] N. G. Thompson, M. Yunovich, and D. Dunmire, "Cost of Corrosion and Corrosion Maintenance Strategies," Corros. Rev., vol. 25, no. 3-4, Jan. 2007, doi: 10.1515/corrrev.2007.25.3-4.247.

[2] L. Kisley, "Single molecule spectroscopy at interfaces," in Spectroscopy and Dynamics of Single Molecules, Elsevier, 2019, pp. 117-161.

[3] W. E. Moerner, "New directions in single-molecule imaging and analysis," Proc. Natl. Acad. Sci., vol. 104, no. 31, pp. 12596-12602, 2007, doi: 10.1073/pnas.0610081104.

[4] T. Chen et al., "Optical Super-Resolution Imaging of Surface Reactions," Chem. Rev., vol. 117, no. 11, pp. 7510-7537, Jun. 2017, doi: 10.1021/acs.chemrev.6b00673.

[5] A. Augustyniak, J. Tsavalas, and W. Ming, "Early Detection of Steel Corrosion via 'Turn-On' Fluorescence in Smart Epoxy Coatings," ACS Appl. Mater. Interfaces, vol. 1, no. 11, pp. 2618-2623, Nov. 2009, doi: 10.1021/am900527s.

[6] A. Saini, H. Messenger, and L. Kisley, "Fluorophores 'turned-On' by Corrosion Reactions Can Be Detected at the Single-Molecule Level,” ACS Appl. Mater. Interfaces, vol. 13, no. 1, pp. 2000-2006, Jan. 2021, doi: 10.1021/acsami.0c18994. 